\title{
Cultivation of Human Chondrocytes on Cartilage Fluff Matrix without Loss of Phenotypic Expression
}

Theodore Malinin* and Billy E Buck

Department of Orthopedics, University of Miami Miller School of Medicine, USA

\begin{abstract}
Chondrocytes grown from cartilage explants and then cultured with rehydrated previously desiccated thin strands of cartilage called "cartilage fluff matrix" or "fluff" filled the gaps between the strands of fluff making new cartilage and invaded the cartilage strands. The cells stained similar to the deep layers of normal articular cartilage though at least 60 days of culture. The chondrocytes binded the cartilage strands together and filled the gaps with new cartilage creating a solid construct.
\end{abstract}

Keywords: Cartilage; Engineering; Chondrocytes; Cartilage fluff; Regeneration; Cell culture; Allogeneic chondrocyte

\section{Introduction}

Numerous attempts have been made in the past to find the means of regenerating articular chondral defects in humans [1]. Transplantation of autologous chondrocytes has been one method to achieve this goal [2]. However, transplantation of autologous chondrocytes had only qualified success because of limited amount of source material and the time required to expand the cells [3].

Allogeneic chondrocyte transplantation had been performed only experimentally, although if such could be achieved successfully in patients, it would obviate many problems encountered with autologous chondrocyte transfers. One of the advantages of allogeneic chondrocyte transplantation would be the availability of adequate material. Consequently the cells would not have to be expanded [1].

The present study was performed to investigate the method of cultivating large numbers of chondrocytes in primary or low passage cultures on a suitable matrix. The hypothesis was that a suitable matrix would allow growth of chondrocytes in large numbers with retention of their phenotypes. To this end we created non- viable cartilage fluff matrix on the premise that it would stimulate cartilage cell growth because of chondrogenic stimuli being inherently present in this tissue. Cartilage fluff is a unique tissue composition made up of thin branching strands of cartilage [4].

\section{Materials and Methods}

Cartilage fluff matrix was prepared from cadaver tissue donors with normal appearing, glistening articular cartilage. Cartilage fluff was prepared by grating articular cartilage into strands from $800 \mu \mathrm{m}$ to $2000 \mu \mathrm{m}$ in length and having an average width of about $50 \mu \mathrm{m}$. The cartilage fluff was preserved by either freeze- drying or hypothermic dehydration. A photograph of cartilage fluff is provided in (Figure 1).

Chondrocyte cultures were initiated from articular cartilage explants. Cells from these explants, as shown in (Figure 2), migrate from the explants and retain morphologic characteristics of mature chondrocytes. Explants of cartilage were placed into $75 \mathrm{~cm}^{2}$ plastic Corning flasks with tissue culture media CMRL 1415 or RPMI 1640 media supplemented with L-glutamine or chondrogenic media (Lifeline cell technology, Frederick, MD) and with $10 \%$ fetal bovine serum. The flasks were maintained in incubators in $5 \% \mathrm{CO}_{2}$ atmosphere at $36^{\circ} \mathrm{C}$. When the cells became confluent the explants were removed from the flasks, and the cells scraped with rubber policemen and placed into a suspension. Cells were seeded directly onto culture wells and allowed to form a monolayer, usually in 7 days.
Cells were seeded into $40 \mathrm{~mm}$ diameter uncoated wells. Six well tissue culture plates with low evaporation lids were used. In half of the plates (N-6) cartilage fluff matrix was placed directly on the monolayer chondrocyte cultures after seven days of cultivation. Alternately cells were compacted into pellets by centrifugation. Chondrocyte pellet were then placed on top of the matrix. In other cultures (N-4) cartilage matrix was placed directly in a well without monolayer cells. Chondrocyte pellet was then placed directly on top of the cartilage matrix. These were grown in chondrogenic differentiation media supplemented with $10 \%$ fetal bovine serum $[5,6]$.

In another iteration, BD Falcon $40 \mu \mathrm{m}$ nylon cell strainers with their handles removed were inserted into a 6 well non adherent cell culture Costar plate. Fluff, chondrogenic media and chondrocytes were added sequentially inside the filter, serving as a basket. The plate was put on a rocker inside the incubator. Media was changed every three or four days up to day 70. Plates with chondrocyte cultures and fluff were

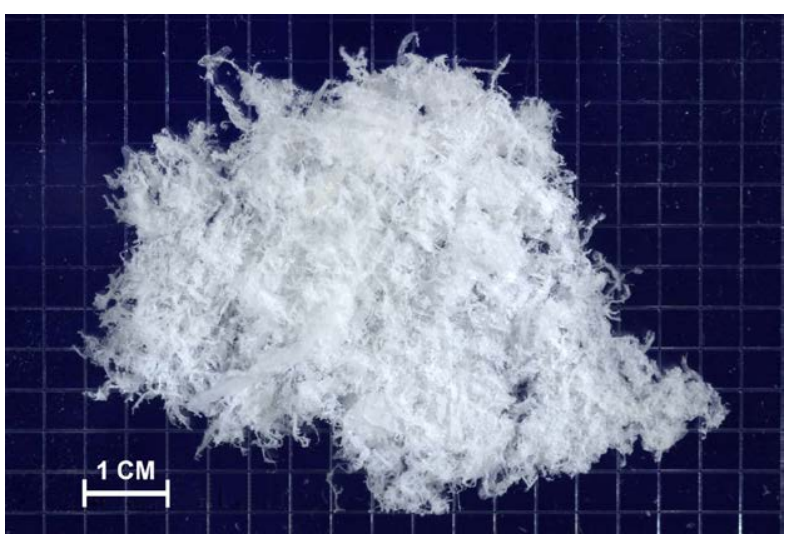

Figure 1: Cartilage fluff matrix

*Corresponding author: Theodore Malinin, Department of Orthopedics, University of Miami Miller School of Medicine, Tissue Bank, 1951 NW 7th Avenue, Suite 200, Miami, FL 33136, USA, Tel: 305-689-1403; E-mail: tmalinin@med.maimi.edu

Received August 20, 2014; Accepted October 14, 2014; Published October 16, 2014

Citation: Malinin T, Buck BE (2014) Cultivation of Human Chondrocytes on Cartilage Fluff Matrix without Loss of Phenotypic Expression. J Transplant Technol Res 4: 138. doi:10.4172/2161-0991.1000138

Copyright: ( 2014 Malinin T, et al. This is an open-access article distributed under the terms of the Creative Commons Attribution License, which permits unrestricted use, distribution, and reproduction in any medium, provided the original author and source are credited. 


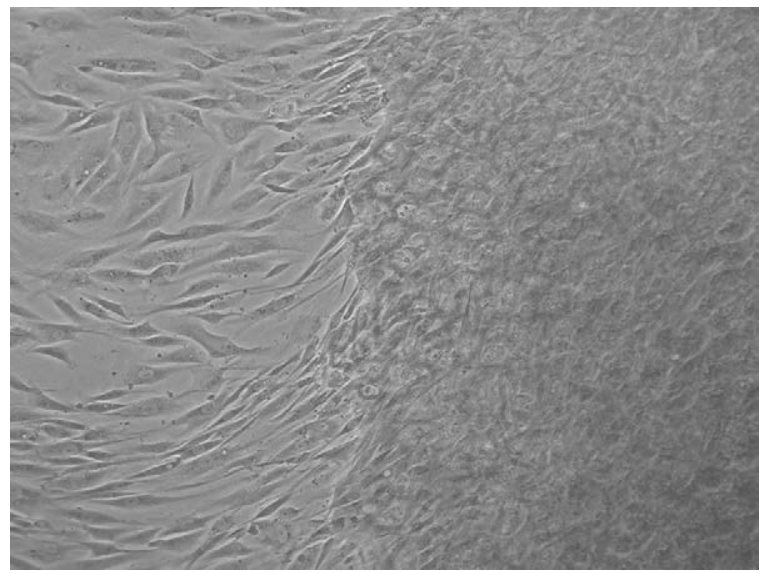

Figure 2: Chondrocytes migrating away from a cartilage explant. Phase contrast. x100.

continuously rocked in the incubator. For control two preparations were used. One consisted of placing cartilage cell pellets on top a layer of microparticulate bone 90 to $300 \mu \mathrm{m}$ in size (N-4). The other consisted of placing periosteal explants on a chondrocyte fluff (N-4). The cultivation technique was the same as that used for chondrocytes on the cartilage fluff.

Cultures were maintained in the dark in $5 \% \mathrm{C}_{2}$ atmosphere in incubators at $36^{\circ} \mathrm{C}$. One experimental culture was sacrificed at 14 days, and one at 40 days. The remaining cultures were fixed at 60 days. One culture was fixed at 70 days.

Cultures were observed in a Nikon inverted phase contrast microscope every 3-4 days. The wells were likewise photographed. At the end of observation period cultures with fluff were fixed in 10\% formalin in Earle's balanced salt solution, dehydrated and embedded in paraffin.

Sections were cut at $5 \mu \mathrm{m}$ and $10 \mu \mathrm{m}$ and stained with hematoxylin and eosin, Romanowski-Giemsa stain, periodic acid Schiff (PAS) stain and safranin $\mathrm{O}$ stain. Sections were viewed in a Leitz Orthoplan microscope. Photographs were obtained using Spot Insight OE digital camera and a Photoshop program for processing images.

\section{Results}

Chondrocytes in contact with cartilage fluff matrix grew vigorously, attached themselves to the cartilage strands, filled the spaces in-between and formed a cartilaginous tissue complex depicted in (Figure 3), as early as after 14 days of incubation.

Microscopically cultured chondrocytes attached themselves to the non-viable cartilage strands and filled in spaces between the strands (Figure 4). Thus the growing cultured cells held the cartilage strands together (Figure 5). As seen in Figure 4 the cells also invaded the cartilage strands and established new chondrocytes within the same, presumably in the pre-existing lacunae (Figure 6). The cells which attached themselves to the edges of the cartilage matrix strands formed palisade arrangements and clearly penetrated into the matrix (Figure 7). These cells were metabolically active. The cytoplasm of these cells contained abundant proteoglycan granules, demonstrated by their PAS positivity (Figure 8) as well as by intense staining with safranin 0 (Figure 9).

Chondrocytes growing in conjunction with cartilage fluff matrix maintained their polyhedral shapes and did not undergo transformation into fibroblast-like cells. They also exhibited intercellular bridges and connections, something we had commonly observed in monolayer chondrocyte cultures (Figure 10).

Chondrocytes in the pellet form placed on top of layers of microparticulate bone 100 to 300 microns in size [7] failed to proliferate. Therefore the cultures were terminated at 30 days. Periosteal explants placed on cartilage fluff exhibited only a minimal growth. These too were sacrificed at 30 days.

\section{Discussion}

We developed a method of cultivating human chondrocytes on human cartilage fluff matrix. This matrix appears to be particularly suitable for the task. This cartilage composition comprising nondemineralized thin branching cartilage strands is a self-supporting matrix containing growth factors which enhance growth of chondrocytes in vitro. During the period of cultivation up to 70 days these did not undergo dedifferentiation and retained their morphologic phenotype. Thus it seems reasonable to postulate the presence of chondrogenic stimulus in this matrix material. This contention is supported by the finding of cartilage microparticles enhancing expression of aggrecan and a decreased expression of collagen $\mathrm{X}$, both at the mRNA and protein levels [8].

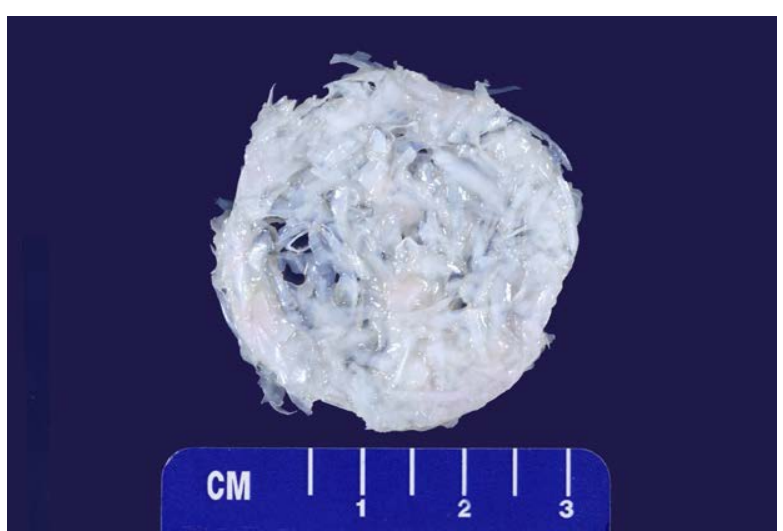

Figure 3: Cartilage fluff matrix with growing chondrocytes, after 70 days in culture.

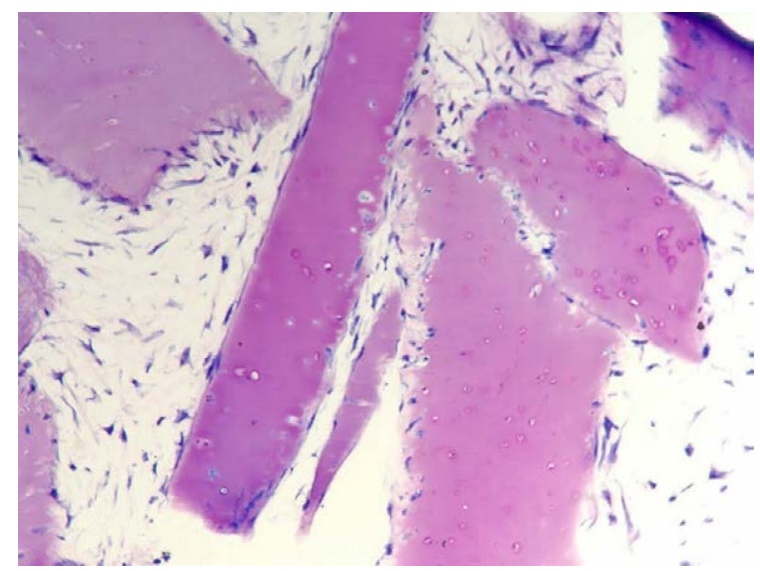

Figure 4: Histological section of cartilage fluff matrix with cultured chondrocytes attached to the strands and growing in between the strands, after 40 days in culture. Cells penetrate and repopulate non- viable cartilage matrix. Romanowski-Giemsa stain. x100. 


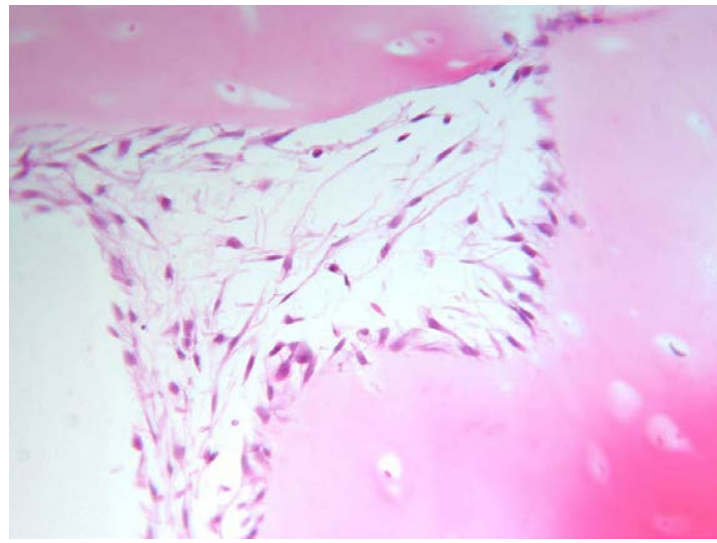

Figure 5: Cultured chondrocytes filling the space between two cartilage strands, after 14 days of cultivation. H\&E $\times 250$.

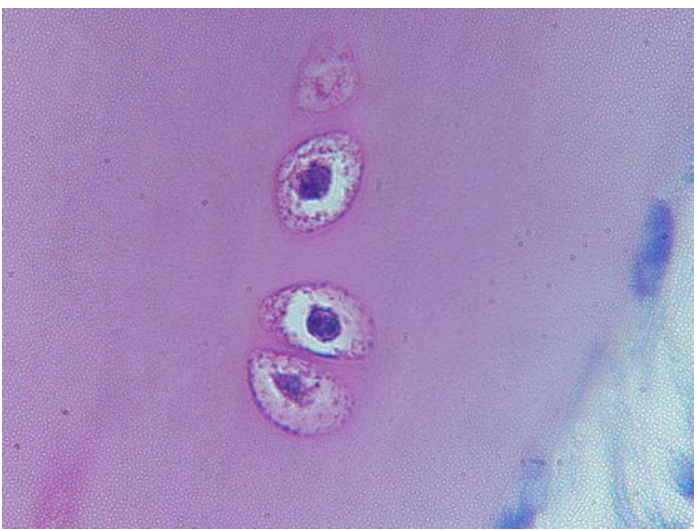

Figure 6: Newly formed chondrocytes in a repopulated cartilage strand. Romanowski-Giemsa stain. x900.

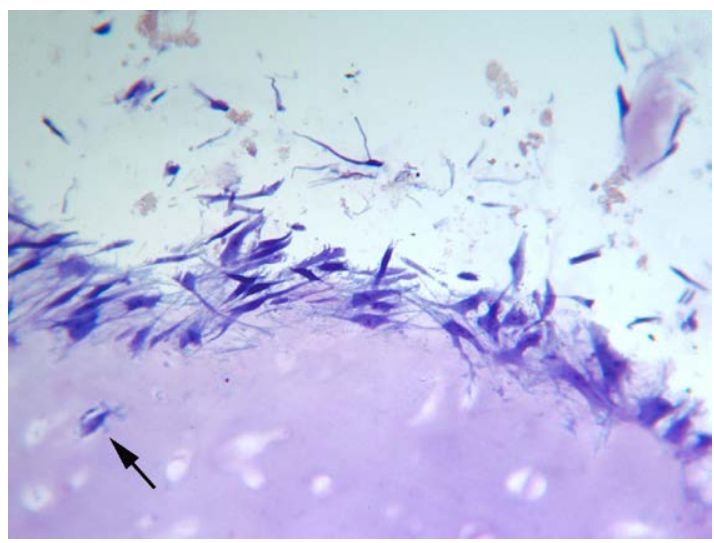

Figure 7: Chondrocytes after 14 days in culture formed a palisade arrangement and penetrated non-viable cartilage matrix. Arrow shows a cell lodged in an empty lacuna. Romanowski-Giemsa stain. x250.

Cultured chondrocytes, maintained their polyhedral shapes, and did not transform into spindle-shaped cells. Furthermore, they bridged the space between the cartilage strands and made new cartilage matrix. The construct thereby eventually became a single coherent mass. It must be born in mind that in $5 \mu \mathrm{m}$ thick sections larger separations between cells were noted as compared to $10 \mu \mathrm{m}$ thick sections.
The cytoplasm of these cells stained blue in Romanowski- Giemsa preparation, red in PAS stained sections and orange red in safranin $\mathrm{O}$ preparations. These tinctorial properties of cells indicated presence of mucopolysaccharides present in normal chondrocytes. The nuclei

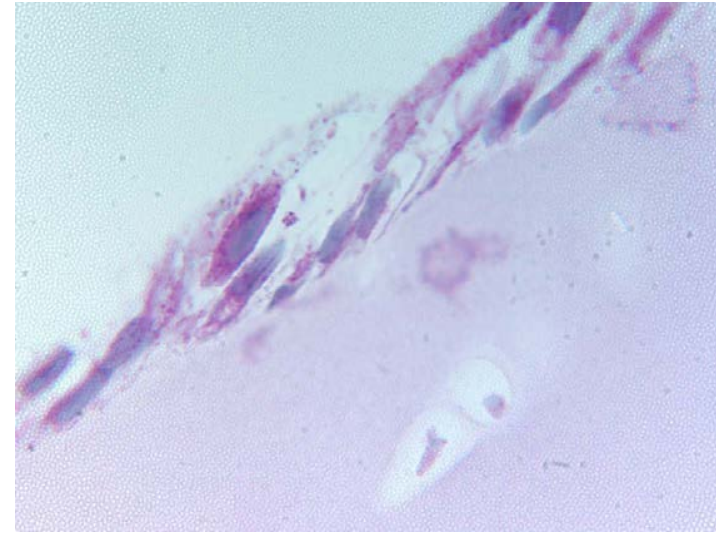

Figure 8: Chondrocytes at the margins of cartilage strands contain abundant PAS positive particles indicating the presence of glycoproteins, glycolipids and polysaccharides. PAS-hematoxylin. $\mathrm{x} 900$.

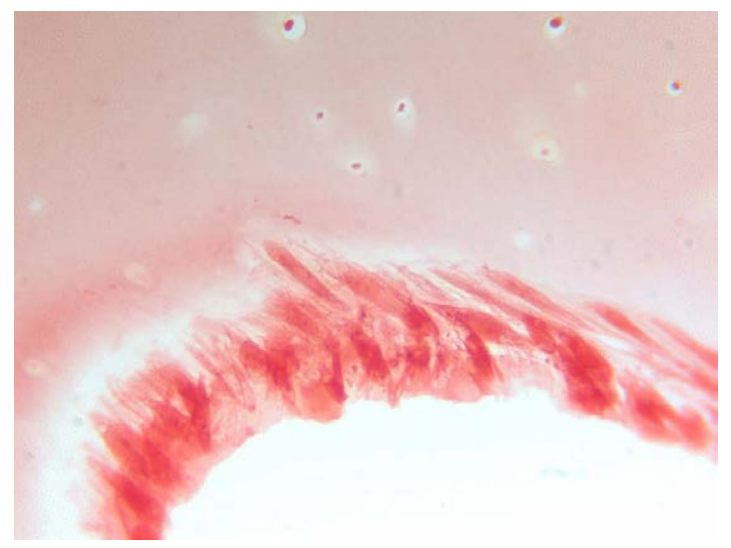

Figure 9: Cells at the cartilage rim and those penetrating the cartilage matrix stain strongly with safranin 0 , indicating presence of cartilage proteoglycans. Safranin 0. x400.

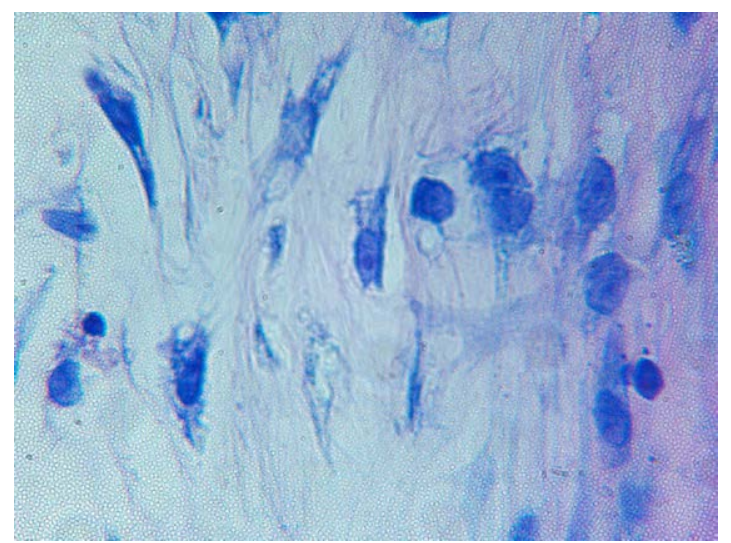

Figure 10: Sheath of chondrocytes growing in between cartilage strands. These cells exhibit the morphological features of chondrocytes in culture including large basophilic nuclei as well as intercellular bridges. RomanowskiGiemsa stain. x900. 
were large and basophilic with distinct nucleoli and denovo synthesis of proteoglycans and glycoproteins. The cell bodies usually stood away from their neighbors but there were intercellular connections, perhaps filopodia or lamillipodia cell projections. Similar observations are common in monolayer cultures [9]. However, in viewing fixed, stained cell preparation one must bear in mind that these are shrunken compared to viable cells viewed in phase-contrast.

Cartilage fluff matrix used in the present experiments was prepared either by freeze-drying or by hypothermic dehydration. The latter method is less destructive than freeze-drying as it avoids alterations produced by freezing and associated phenomena such as "collapse" which occurs in freeze-dried materials.

Given the findings presented, it seems reasonable to speculate that these in-vitro findings represent two phenomena. One is that proliferating chondrocytes migrate into vacant lacunae within the cartilage strands and take up their role in maintaining the nearby cartilage matrix. The second is that the proliferating chondrocytes between the strands begin to form extracellular collagen matrix.

The failure of the chondrocytes to grow on the layer of microparticulate bone maybe attributed to several factors. However, the most plausible explanation can be based on the inherent properties of the chondrocytes themselves. Hyaline cartilage consists of chondrocytes and structurally non-uniform extracellular matrix (ECM). Their properties define the sum total of hyaline cartilage morphology [10]. Thus both of these cellular and acellular components, jointly form a single integrated entity. Cartilage collagens serve as meshwork for proteoglycans (PG's) and provide skeleton of the integrated entity $[10,11]$. In the present setting viable chondrocytes and non-viable cartilage matrix complimented each other by forming a unique structural unit whereby chondrocytes acted as truly architects of new cartilage and interacted with non- viable cartilage matrix strands to produce a new structure.

As far as periosteum growing poorly or not at all on cartilage fluff, the explanation remains elusive. Cultured periosteal cells are known for their potential of osteogenic differentiation [13]. Periosteum also contains chondrogenic progenitor cells [14]. However, thus far periosteal cells were used primarily for bone and not cartilage regeneration. A reasonable explanation for the failure of periosteal explants to produce cartilage, in our system may well be that interaction between chondrocytes and cartilage matrix is required to grow new cartilage.

Currently several problems are encountered in the treatment of articular chondral and osteochondral and defects. Transplantation of allogeneic chondrocytes may alleviate some of these problems. Unlike with autologous chondrocytes, there is no limitation of the quantity of allogeneic chondrocytes which would be available for transplantation. In this connection retention of the original phenotype is important. The technique described in this report might facilitate development of a tissue-engineered entity suitable for transplantation and repair of diseased articular hyaline cartilage. Although the present work is only in the initial stages, hopefully it will encourage further developments in this direction.

\section{References}

1. Malinin TI, Temple HT (2013) Musculoskeletal Tissue Transplantation and Tissue Banking. Jaypee Brothers Medical Publishers 1: 132-143.

2. Marlovits S (2008) Autologous chondrocyte cartilage repair. Musculoskeletal Tissue Regeneration 369-394.

3. Niemeyer P, Pestka JM, Kreuz PC, Erggelet C, Schmal H, et al. (2008) Characteristic complications after autologous chondrocyte implantation for cartilage defects of the knee joint. Am J Sports Med 36: 2091-2099.

4. US Patent \# 8,791,071

5. Chen CW, Tsai YH, Deng WP, Shih SN, Fang CL, et al. (2005) Type I and II collagen regulation of chondrogenic differentiation by mesenchymal progenitor cells. J Orthop Res 23: 446-453.

6. Wise JK, Yarin AL, Megaridis CM, Cho M (2009) Chondrogenic differentiation of human mesenchymal stem cells on oriented nanofibrous scaffolds: Engineering the Superficial Zone of Articular Cartilage. Tissue Eng Part A 15: 913- 921.

7. Malinin TI, Carpenter EM, Temple HT (2007) Particulate bone allograft incorporation in regeneration of osseous defects; importance of particle sizes. Open Orthop J 1: 19-24.

8. Delcroix GJ (2015) MIAMI Cell hyaline cartilage formation is enhanced by TGF- B3-releasing pharmacologically active microcarriers and human cartilage microparticles. Abstract 2015 annual meeting, Orthopaedic Research Society.

9. Malinin TI, Buck BE, Temple HT (2009) Cell to cell contacts between articula cartilage chondrocytes in cell culture. 8th World congress, International Cartilage Repair Society.

10. Malinin GI, Malinin TI (1999) Microscopic and histochemical manifestations of hyaline cartilage dynamics. Prog Histochem Cytochem 34: 163-242.

11. Burgeson RE, Nimni ME (1992) Collagen types. Molecular structure and tissue distribution. Clin Orthop Relat Res : 250-272.

12. van der Rest M, Garrone R (1991) Collagen family of proteins. FASEB J 5 2814-2823.

13. Redlich A, Perka C, Schultz O, Spitzer R, Häupl T, et al. (1999) Bone engineering on the basis of periosteal cells cultured in polymer fleeces. J Mater Sci Mater Med 10: 767-772.

14. Tornberg DN, Bassett CA (1977) Activation of the resting periosteum. Clin Orthop Relat Res : 305-312. 